\title{
The first insights into regulation of cell transdifferentiation during gut regeneration in Eupentacta fraudatrix
}

\author{
Alexey Boyko \\ NSCMB FEB RAS, Vladivostok, Russia \\ Alteroldis@gmail.com
}

\begin{abstract}
The holothurian Eupentacta fraudatrix is a unique organism for studying regeneration mechanisms. It has been repeatedly confirmed that regeneration is only due to the transformation of terminally differentiated cells. In this study, we examined changes in gene expression during gut regeneration of the holothurian E. fraudatrix and found $11 \mathrm{TFs}$, which are candidates for the role of transdifferentiation regulators
\end{abstract}

Keywords - echinoderms, transdifferentiation, gene ontology, gene expression, RNA-seq

\section{Motivation and aim}

Motivation

It is well-known that regeneration in E. fraudatrix is only due to the transformation of terminally differentiated cells. In addition, at the histological level, this process has been well studied previously. However, the molecular basis of cell transdifferentiation mechanisms in E. fraudatrix remains unknown.

Aim

The morphological features of regeneration of internal organs and cell transdifferentiation in the holothurian $E$. fraudatrix have been well studied [1]. In order to understand, to a first approximation, the possible molecular processes that occur during transdifferentiation in holothurians, we decided to use RNA-seq. In this work, we tried to obtain the complete transcriptome and determine the differential expressed genes (DEGs) at the stage of active cell transdifferentiation and transcription factors (TFs), which are candidates for the role of transdifferentiation regulator.

\section{Methods}

Tissue of gut was sampled from the intact gut and on the third (first stage of regeneration), fifth-seventh (second stage), and tenth (third stage) days post-evisceration. Each sample was sequenced and represented in two biological replicates with 5 individuals, pooled together, per replicate. Then the clean reads were corrected and assembled using SPAdes 3.13 software and our own Python script as described in the article [2]. Annotation was carried out by NR NCBI, Echinobase and Human Ensemble protein databases. Orthologs of human proteins were identified using a custom Python script that implements modified reciprocal method for finding the best hit. To identify the most likely candidates for the role of transdifferentiation regulators, only the TFs satisfying the three conditions were taken into consideration: the values of TPMs (Transcripts Per Kilobase Million) of the second stage higher than unity; the average value of LogFC (logarithm of fold change with base 2) of the second stage relative to the first and third stages more than half; the value of LogFC (logarithm of fold change) of the second stage relative to the first or third stages more than unity. The differential expression was evaluated for the sequences using Bowtie v2.3.4, RSEM v1.3.1 and DESeq2 v1.18 software. The enrichment analysis of biological processes and pathways was performed with GSEA software in accordance with the EnrichmentMap protocol for RNA-seq data [3]. Only processes or pathways containing at least one of the 11 most likely candidates for the role of transdifferentiation regulators were used. Then, results of the enrichment analysis were visualized using EnrichmentMap plug-ins in Cytoscape software

\section{Results}

Transcriptomes of intestinal anlage of the three stages of regeneration, as well as the normal gut, were sequenced with an Illumina sequencer (San Diego, CA, USA). We identified 14,617 sea urchin protein homologs, of which 308 were transcription factors. After analysing the dynamics of gene expression during regeneration and the map of biological processes in which they participate, we identified 11 factors: Ef-EGR1, Ef-ELF, Ef-GATA3, Ef-ID2, Ef-KLF1/2/4, EfMSC, Ef-PCGF2, Ef-PRDM9, Ef-SNAI2, Ef-TBX20, and EfTCF24. With the exception of TCF24, they are all involved in the regeneration, development, epithelial-mesenchymal transition, and immune response in other animals. We suggest that these transcription factors may also be involved in the transdifferentiation of coelomic epithelial cells into enterocytes in holothurians.

\section{ACKNOWLEDGMENT}

The results were obtained using the equipment of CKP «Primorsky aquarium», National Scientific Center of Marine Biology FEB RAS (Vladivostok, Russia). This study was supported by the Russian Foundation for Basic Research (grant No. 19-34-90015).

\section{REFERENCES}

[1] Mashanov V.S. et al. (2005) Transdifferentiation in holothurian gut regeneration. Biol. Bull. 209: 184-193.

[2] Boyko A.V. et al. (2019) Reference assembly and gene expression analysis of Apostichopus japonicus larval development. Sci. Rep. 9: 111.

[3] Merico D. et al. (2010) Enrichment Map: a network-based method for gene-set enrichment visualization and interpretation. PLoS One 5: e13984 